\title{
CANADA: MODÈLE D'ÉTAT MULTICULTUREL POUR LE MONDE?
}

\author{
Claude Couture
}

Après l'échec de l'Accord du Lac Meech ${ }^{1}$ en 1990, le gouvernement conservateur de l'époque mit sur pied une vaste consultation populaire pour mieux comprendre l'opinion des Canadiens au sujet des réformes constitutionnelles. Le Forum des citoyens ${ }^{2}$, présidé par Keith Spicer, rencontra près de 400000 Canadiens, visita 42 universités et collèges, reçut 10000 lettres et 75000 appels téléphoniques. Au Québec, lancée en décembre 1990, la commission Bélanger-Campeau reçut 200 mémoires et 600 soumissions ${ }^{3}$. D'autres formes de consultations furent organisées. Par exemple, toujours en décembre 1990, le sous-comité conjoint Sénat-Chambre des communes sur la formule d'amendement à la Constitution commença ses travaux un mois avant la présentation du rapport Allaire au Québec en janvier 1991. En septembre de la même année, le Comité parlementaire sur la Constitution de Dorothy Dobbie et Claude Castonguay (remplacé par Gérard Beaudoin) fut aussi formé et soumis un rapport le 28 février $1992^{4}$. Toutes ces consultations menèrent aux longues négociations de 1992 et finalement à l'Accord de

3 juin 1987 (texte juridique signé par les premiers ministres à Langevin Block, Ottawa), en ligne: UNI $<$ http://www.uni.ca/meech.html $>$.

2 Le Forum des citoyens sur l'avenir du Canada, Rapport à la population et au gouvernement $d u$ Canada, Ottawa, Approvisionnements et Services Canada, 1991.

3 Ottawa, Les rapports Bélanger-Campeau et Allaire, Bibliothèque du Parlement, Service de recherche, BP-257F, 1991.

$4 \quad$ Un Canada renouvelé : rapport du Comité mixte spécial du Sénat et de la Chambre des communes, Ottawa, Imprimeur de la Reine, 1992.
Charlottetown ${ }^{5}$ de juillet-août 1992. D'une certaine façon, aucun autre pays du monde occidental ne fut ainsi mobilisé de façon démocratique et non violente pour en arriver à un nouveau contrat constitutionnel. Les principales dimensions de ces discussions de 1990 à 1992 avaient été la question des relations anglophonesfrancophones, la reconnaissance des Premières Nations et la place du multiculturalisme. Aussi, aucun autre pays du monde occidental n'a été aussi loin dans la représentation symbolique de ces dimensions dans ses institutions constitutionnelles. En ce sens, le multiculturalisme canadien, qui ne peut être séparé des autres dimensions, est unique. Pour expliquer ce phénomène, nous proposons un texte en trois parties : d'abord un rappel du contexte créé pour les institutions de l'ère Trudeau; ensuite un résumé des débats américains et canadiens sur les origines idéologiques du Canada et, en particulier, l'apport récent dans ce débat de l'historien Gérard Bouchard; enfin, selon la perspective de ces débats, il sera proposé une interprétation du processus menant à Charlottetown et de la clause Canada de l'accord de 1992, deux indices qui montrent le caractère unique de la reconnaissance de la diversité au Canada.

\section{LE CONTEXTE}

Meech et Charlottetown ont été dans le prolongement direct des questions sans réponses laissées par les institutions de 1982. Ces

\footnotetext{
Voir en ligne: le Gouvernement du Canada < http://www.pcobcp.gc.ca/aia/default.asp? Language $=\mathrm{F} \&$ Page $=$ consfile $\&$ Sub $=$ TheHistoryofConstitution \&Doc $=$ charlottetown_f.htm $>$.
} 
institutions, la Charte $^{6}$ en particulier, avaient en effet été instaurées au nom d'une logique des droits individuels, alors qu'en fait, les principales dimensions de la Charte reposaient sur un équilibre complexe entre les droits individuels et les droits collectifs. Il importe donc de rappeler brièvement le contexte de ces principales dimensions et leur ambiguïté .

\section{LA LOI SUR LES LANGUES OFFICIELLES}

La question linguistique fut évidemment récurrente dans l'histoire du Canada. Dès 1839, dans son célèbre rapport, Lord Durham avait conclu à l'impossibilité d'une cohabitation de deux communautés linguistiques au sein d'un même État ${ }^{8}$. Ainsi, dans l'Acte d'Union, $1840^{9}$, l'article 41 stipulait l'unilinguisme anglais à la Législature. Toutefois, la victoire des réformistes de Lafontaine-Baldwin et l'obtention du gouvernement responsable en 1848 furent deux facteurs qui eurent comme résultat l'abrogation de cet article et la reconnaissance subséquente du français. Plus tard, l'article 133 de la Loi constitutionnelle de $1867^{10}$ garantissait l'égalité du français et de l'anglais en ce qui concernait les activités du Parlement fédéral et les tribunaux fédéraux. Le même principe s'appliquait à la province du Québec et, à partir de 1870, à la province du Manitoba. Cet article 133, de même que l'article 93 sur l'éducation, contribuèrent à créer cette perception au Québec d'un «pacte» entre les nations ou deux principales communautés linguistiques, du moins en termes numériques, en 1867.

Cette perception, qu'importe qu'elle ait été justifiée ou non, fut assez brutalement démentie par les événements des années 1885 à 1913 : la

$6 \quad$ Charte canadienne des droits et libertés, partie I de la Loi constitutionnelle de 1982, constituant l'annexe B de la Loi de 1982 sur le Canada (R.-U.), 1982, c. 11 [Charte].

7 Pour cette section sur le contexte, voir Claude Couture, $L a$ loyauté d'un lä̈c : Pierre Elliott Trudeau et le libéralisme canadien, Paris, Harmattan, 1996 [Couture, «La loyauté»].

8 Le rapport Durham, trad. par Denis Bertrand et Albert Desbiens, Montréal, Éditions Sainte-Marie, 1969.

9 (R.-U.), 3-4 Vict., c. 35, reproduite dans L.R.C. 1985, app. II, $n^{0} 4$.

10 (R.-U.), 30 \& 31 Vict., c. 3, reproduite dans L.R.C. 1985, app. II, $\mathrm{n}^{0} 5$ [Constitution, 1867]. seconde rébellion des Métis, la pendaison de Riel, l'abolition $\mathrm{du}$ français et des écoles confessionnelles au Manitoba en 1890, l'absence de reconnaissance explicite de l'égalité du français et de l'anglais lors de la création de l'Alberta et de la Saskatchewan et le règlement 17 en Ontario, bref des événements qui ont sérieusement ébranlé l'idée, du moins chez les francophones au pays, que les Canadiens français et les Canadiens anglais puissent vivre côte à côte sans menace pour leurs institutions et leur langue. Ainsi, une certaine asymétrie dans la reconnaissance de l'égalité du français et de l'anglais a-t-elle provoqué chez plusieurs Canadiens français du Québec un changement de perception quant au « pacte ». Or, pour sa part, Pierre E. Trudeau, proposa une société juste. Dans Les années Trudeau, l'ancien rédacteur de Cité libre définit ainsi l'objectif de la société juste :

Dans une telle conjoncture, ce qui m'attira vers la politique, ce n'était plus le désir de lutter pour la liberté, celle-ci étant en quelque sorte devenue le combat d'hier. Dans mon esprit, la valeur à privilégier dans la poursuite de la société juste était plutôt l'égalité. Non pas l'égalité à la Procuste bien sûr, où tous seraient ramenés à une certaine moyenne. Mais l'égalité des chances.[...] Or le Canada me semblait un pays béni des dieux pour poursuivre une politique de la plus grande égalité des chances. Pays jeune, pays riche, pays utilisant deux langues, pays pluraliste par les ethnies et les religions, pays immense à régions géographiques diverses, pays à forme fédérative, le Canada avait de plus une tradition politique ni complètement libertaire ni complètement étatiste, mais qui au contraire reposait sur la collaboration nécessaire des gouvernements avec le secteur privé, et sur l'action directe de l'État pour protéger les faibles contre les 
forts, les démunis contre les biens nantis ${ }^{11}$.

Ainsi, la Loi sur les langues officielles de $1969^{12}$, votée à la suite des recommandations de la Commission sur le bilinguisme et le biculturalisme, visa à corriger une asymétrie évidente quant à l'application de l'article 133 de la Constitution, 1867. Mais pour Trudeau, il s'agissait de créer un climat de justice pour la langue française librement choisie par des individus et non de protéger une communauté. La Loi sur les langues officielles, l'un des fleurons de la politique trudeauiste, a eu un impact évident, surtout hors Québec; ne pas le reconnaître relève de la mauvaise foi. Selon un rapport du Conseil du Trésor pour 1995-96, $29 \%$ des postes dans la fonction publique fédérale étaient occupés par des francophones ${ }^{13}$. Par ailleurs le nombre de postes bilingues depuis 1969 est passé à $31 \%$, un sommet ${ }^{14}$. Ainsi, en rectifiant ainsi près d'un siècle de non-application de l'article 133, le gouvernement de Pierre E. Trudeau, malgré la rhétorique sur les droits individuels, se trouva de facto à appliquer une politique d'action positive en vue de redresser une situation institutionnelle qui marginalisait les Canadiens français. En d'autres mots, par cette politique, on chercha à créer un espace institutionnel collectif favorable à l'épanouissement des francophones. D'où l'idée que la notion de droits collectifs, en équilibre avec celle des droits individuels, était déjà présente dans la Loi sur les langues officielles de 1969. En somme, la première intervention fondamentale de Pierre E. Trudeau en tant que politicien impliqua non pas une opposition entre droits individuels et droits collectifs, mais un subtil équilibre entre ces deux notions. Cet aspect se retrouva dans d'autres politiques, qui doivent être mises en relation avec la question des droits linguistiques.

11 Pierre Elliott Trudeau «Des valeurs d'une société juste», dans Thomas S. Axworthy et Pierre Elliot Trudeau, dir., Les années Trudeau. La recherche d'une société juste, Montréal, Le Jour, 1990, 381 à la p. 382. Nous avons déjà développé cette analyse des institutions de l'ère Trudeau dans Couture, «La loyauté», supra note 7.

12 L.C. $1968-1969$, c. 54

13 «La fonction publique fédérale n'a jamais été aussi francophone et bilingue», Le Franco (11 octobre 1996) 1.

14 Ibid.

\section{LE MULTICULTURALISME}

Depuis les années 1960, le multiculturalisme est devenu l'une des dimensions politiques essentielles du Canada ${ }^{15}$. Le multiculturalisme fit écho à la problématique du biculturalisme des années 1960 et aux débats concernant les relations entre Canadiens anglais et français. La présence d'une population française et catholique au Canada a posé d'emblée, en 1867, la question de la différence dans une société à majorité anglophone et protestante. Près d'un siècle après la Confédération, les tensions entre les Canadiens français et les Canadiens anglais firent craindre aux Canadiens d'origines diverses, ni française, ni anglo-américaine, mais de plus en plus nombreux, qu'une composante essentielle de la société canadienne pourrait être oubliée, voire marginalisée. Le groupe le plus actif, dans les années 1960, fut celui des Ukrainiens qui réclamèrent la reconnaissance d'une " troisième force $»^{16}$. Ainsi, le volume IV de la Commission Laurendeau-Dunton traita de la question de l'apport culturel des « autres Canadiens ${ }^{17}$. Seize recommandations, à partir des données contenues dans ce volume, furent présentées par les commissaires. Et le 8 octobre 1971, Pierre E. Trudeau annonça l'intention du gouvernement fédéral de se lancer dans la promotion du multiculturalisme. Il s'exprima en ces termes :

Aux yeux de la Commission, du gouvernement et, j'en suis sûr, de tous les Canadiens, il ne peut y avoir une politique

15 Pour cette section, voir aussi Yasmeen Abu-Laban, «Liberalism, Multiculturalism and the Problem of Essentialism » (2002) 6:4 Citizenship Studies 459; et, Richard J.F. Day, Multiculturalism and The History of Canadian Diversity, Toronto, University of Toronto Press, 2000; Ian Angus, «Cultural Plurality and Democracy» (2002) 25 International Journal of Canadian Studies 69; Richard Day, «Can there be a Postcolonial Multiculturalism? A Response to Ian Angus» (2002) 26 International Journal of Canadian Studies 127.

16 Yasmeen Abu-Laban, «The Politics of Race and Ethnicity: Multiculturalism as a Contested Arena» dans James P. Bickerton et Alain-G. Gagnon, dir., Canadian Politics, $2^{\circ}$ éd., Petersborough, Broadview Press, 1994, 242; Voir aussi Yasmeen Abu-Laban et Christina Gabriel, Selling Diversity, Peterborough, Broadview Press, 2002.

17 Canada, Rapport de la Commission royale d'enquête sur le bilinguisme et le biculturalisme, vol. IV, Ottawa, Imprimeur de la Reine, 1967. 
culturelle pour les Canadiens d'origine française et britannique, une autre pour les autochtones et encore une pour tous les autres. Car, bien qu'il y ait deux langues officielles, il n'y a pas de culture officielle, et aucun groupe ethnique n'a la préséance. Il n'y a pas un citoyen, pas un groupe de citoyens qui soit autre que canadien, et tous doivent être traités équitablement.

La Commission était persuadée que l'adhésion à un groupe ethnique ne dépend pas tellement de l'origine ou de la langue maternelle, mais du sentiment d'appartenir au groupe et de ce que la Commission appelle « la volonté collective du groupe d'exister ». Le gouvernement partage ce point de vue.

Tout homme verrait sa liberté entravée s'il se trouvait enfermé pour toujours dans un compartiment culturel déterminé uniquement par sa naissance ou sa langue. Il est donc essentiel que tout Canadien, quelle que soit son origine ethnique, puisse apprendre au moins l'une des deux langues dans lesquelles le pays conduit les affaires publiques.

Le multiculturalisme dans un cadre bilingue apparaît au gouvernement comme le meilleur moyen de préserver la liberté culturelle des Canadiens. Une politique de ce genre devrait permettre de réduire la discrimination et la jalousie qu'engendrent les différences de culture. Pour que l'unité nationale ait une portée personnelle profonde, il faut qu'elle repose sur le sens que chacun doit avoir de sa propre identité; c'est ainsi que peuvent naître le respect pour les autres, et le désir de partager des idées, des façons de voir. Une politique dynamique de multiculturalisme nous aidera à créer confiance en soi qui pourrait être le fondement d'une société où règnerait une même justice pour tous [...].
En appliquant sa politique de multiculturalisme dans un cadre bilingue, le gouvernement apportera son aide de quatre façons différentes :

Premièrement, compte tenu de ses ressources, le gouvernement s'efforcera d'aider tous les groupes culturels canadiens qui ont manifesté le désir et la volonté de développer la capacité de s'accroître et d'ajouter à la vie canadienne, et dont le besoin d'assistance est évident, qu'il s'agisse de petits groupes faibles ou de groupes importants et fortement organisés.

Deuxièmement, le gouvernement aidera les membres de tout groupe culturel à surmonter les barrières culturelles qui les empêchent de participer pleinement à la société canadienne.

Troisièmement, le gouvernement favorisera les rencontres et échanges entre tous les groupes culturels du Canada dans l'intérêt de l'unité nationale.

Quatrièmement, le gouvernement continuera d'aider les immigrants à apprendre au moins l'une des deux langues officielles et à s'intégrer entièrement dans la société canadienne ${ }^{18}$.

On remarquera encore une fois que cet énoncé de politique renvoie à des catégories collectives. Dans la mesure en effet où le multiculturalisme devait s'inscrire dans un cadre bilingue, le principe fut manifestement de favoriser l'intégration harmonieuse de groupes de diverses origines au sein de l'une ou de l'autre (ou des deux à la fois) communautés nationales et linguistiques d'origine européenne. La promotion du multiculturalisme dans le cadre du bilinguisme impliquait forcément la reconnaissance de différences de contextes pour des individus provenant de cultures et de groupes

18 Débats de la Chambre des communes, (8 octobre 1971) à la p. 8546 . 
différents. Là encore, il ne semble y avoir eu aucune opposition entre, à tout le moins, la notion de collectivité et celle de droits individuels.

\section{Le LiVRe Blanc sur les Autochtones}

Dans le but, sans doute, d'atteindre encore une fois les objectifs d'une société juste, dans le sens d'une égalité des citoyens, le gouvernement de Pierre E. Trudeau présenta en 1969 un Livre Blanc sur la question autochtone. Le Livre Blanc proposait l'abolition graduelle du ministère des Affaires indiennes et de la Loi sur les Indiens ${ }^{19}$ dans un délai de cinq ans. On prévoyait également l'élimination du statut d'Indien. Or, ce programme fit peu de cas des revendications autochtones, notamment la reconnaissance des traités et la légitimité de certaines revendications territoriales.

Ainsi, depuis la fin du $\mathrm{XIX}^{\mathrm{e}}$ siècle, les Autochtones s'étaient regroupés en diverses associations $^{20}$. Les Nisga'a en particulier furent très militants en Colombie-Britannique, et ce, dès 1890. Par la suite, deux autres associations furent formées, les Tribus alliées de ColombieBritannique, en 1915, et la Fraternité des Autochtones de la Colombie-Britannique, en 1931. En Ontario et au Québec, l'une des premières associations, formée en 1918, fut la Ligue des Indiens du Canada. À partir de la Seconde Guerre mondiale, les associations proliférèrent : en 1939, l'Association des Indiens de l'Alberta; en 1944, les Indiens de la Saskatchewan; en 1943, la Fraternité des Indiens d'Amérique du Nord ${ }^{21}$.

Face aux revendications des Autochtones, deux Commissions d'enquête furent formées, la première en 1946-48, et la seconde en 1959-61. Les travaux de ces deux commissions eurent comme résultat de susciter une nouvelle vague de militantisme. En 1960 au niveau fédéral, en 1965 en Alberta et en 1969 au Québec, le droit de vote fut accordé aux Autochtones. Ce fut donc dans ce contexte de militantisme que le gouvernement Trudeau présenta son Livre Blanc. Ce document,

19 L.R.C. 1985 , c. I-5.

20 Olive Patricia Dickason, Canada's First Nations: A History of Founding Peoples From Earliest Times, Toronto, McClelland and Stewart, 1992.

21 Ibid. nous l'avons déjà fait remarquer, fut reçu avec beaucoup d'hostilité. Ce qui, cependant, fit reculer le gouvernement, fut la décision de la Cour suprême du Canada dans l'affaire Calder en $1973^{22}$. Malgré le vote en défaveur de la nation Nisga'a, le principe des titres de propriété autochtones établis en fonction d'une réinterprétation de la Proclamation royale de 1763 fut reconnu. L'année suivante, un Bureau des revendications autochtones entreprit la lourde tâche de traiter les revendications territoriales autochtones. Ensuite, il fut reconnu en 1982, dans la Loi constitutionnelle, plus précisément dans la Charte, à l'article 25, et dans l'article 35 de la Loi, le principe des droits des peuples autochtones du Canada, notamment les droits ancestraux ou issus de traités ${ }^{23}$. L'exemple autochtone fit apparaître, une fois de plus, qu'on ne peut dissocier les individus de leur contexte spécifique.

De fait, le point culminant de la carrière de Pierre E. Trudeau fut évidemment le rapatriement de la Constitution canadienne et l'inclusion d'une charte des droits et liberté. Or nous avons déjà fait remarquer que dans la Charte, les droits collectifs des Autochtones sont reconnus par l'article 25. Mais ce n'est pas le seul article à caractère collectif. L'article 15 sur le droit à l'égalité, notamment l'égalité selon le sexe, les articles 16 à 22 , sur les langues officielles, l'article 23 sur les droits à l'instruction dans la langue de la minorité, constituent une forme de protection pour les individus faisant partie de collectivités précises. Ainsi, la Charte constitua un délicat exercice d'équilibre entre les articles s'inscrivant dans une stricte perspective des droits individuels (articles 1 à 15) et les articles que nous venons d'énumérer.

On voit donc que les institutions de l'ère Trudeau ont subtilement articulé les notions de droits collectifs et de droits individuels. Une telle articulation n'est certainement pas incompatible avec les théories contemporaines de la justice. Charles Taylor et James Tully ont bien fait ressortir l'importance de situer la question des droits individuels dans des contextes spécifiques,

Calder c. Procureur général de la Colombie-Britannique, [1973] R.C.S. 313.

23 Gerald R. Alfred, Heeding the Voices of' Our Ancestors, Toronto, Oxford University Press, 1995. 
$\operatorname{concrets}^{24}$.

En 1971, John Rawls publia justement une Théorie de la justice par laquelle il chercha à définir des institutions sociales qui garantiraient un équilibre dans la satisfaction des aspirations des individus ${ }^{25}$. Aucun groupe ne devrait avoir un avantage moral à long terme sur un autre. À partir de ce point de départ, nous devons construire un contrat social dans lequel on retrouverait une sincère inquiétude quant au bien-être de chacun, en raisonnant à partir de l'élimination systématique, face à différentes possibilités, de la plus répréhensible ${ }^{26}$.

Les principes ici sont donc : 1) que chaque individu a le droit à la plus grande liberté possible si elle est compatible avec la liberté des autres; 2) que les inégalités sociales doivent être confrontées à l'ouverture de postes et de places dans des conditions qui favorisent une égale opportunité; 3 ) les inégalités sont temporairement justifiées si elles permettent d'éviter, à court terme, des situations pires. Ainsi, la persécution, la discrimination et l'oppression politique sont

24 James Tully, Strange Multiplicity: Constitutionalism in an Age of Diversity, Cambridge, Cambridge University Press, 1995; Charles Taylor, Rapproche les solitudes, Québec, Presses de l'Université Laval, 1992. Voir aussi Guy Laforest, De la prudence, Montréal, Boréal,1993.

25 Dans $A$ Theory of Justice, Cambridge, Cambridge University Press, 1971 à la p. 507, John Rawls a notamment écrit :

" This account of the basis of equality calls for a few comments. First of all, it may be objected that equality cannot rest on natural attributes. There is no natural feature with respect to which all human beings are equal, that is, which everyone has (or which sufficiently many have) to the same degree. It might appear that if we wish to hold a doctrine of equality, we must interpret it in another way, namely as a purely procedural principle. Thus to say that human beings are equal is to say that none have a claim to preferential treatment in the absence of compelling reasons. The burden of proof favors equality : it defines a procedural presumption that persons are to be treated alike. Departures from equal treatment are in each case to be defended and judged impartially by the same system of principles that hold for all; the essential equality is thought to be equality of consideration $»$.

26 Ibid. à la p. 242, Rawls a aussi écrit: « Viewing the situation from the legislative stage, one may decide that the formation of paramilitary groups, which the passing of the statute may forestall, is a much greater danger to the freedom of the average citizen than being held strictly liable for the possession of weapons. Citizens may affirm the law as the lesser of two evils, resigning themselves to the fact that while they may be held guilty for things they have not done, the risks to their liberty on any other course would be worse. Since bitter dissensions exist, there is no way to prevent some injustices, as we ordinarily think of them, from occurring. All that can be done is to limit these injustices in the least unjust way ». incompatibles avec la liberté « égale». Par ailleurs la règle de l'opportunité égale garantit une égalité des chances de réussite, peu importe la classe sociale d'origine. En somme, certaines théories libérales contemporaines, même lorsque le point de départ se trouve sans référence aux origines sociales ou autres, the veil of ignorance, aboutissent à une reconnaissance de la situation concrète des individus, donc de leur contexte dans une collectivité ${ }^{27}$. En ce sens, Meech et Charlottetown ont été dans le prolongement direct de ce que 1982 avait ouvert comme possibilités.

\section{Meech et Charlottetown}

Nous n'entrerons pas dans les détails d'une saga constitutionnelle qui mobilisa pendant plusieurs années toute une société. Remarquons toutefois que très peu de sociétés, voire aucune, comme nous l'avons fait remarquer en introduction, ont été ainsi mobilisées de façon démocratique, sans guerre civile, pour discuter de la refonte du contrat social et politique fondamental du pays. Aux deux accords dits du lac Meech et de Charlottetown, Pierre E. Trudeau s'opposa cependant avec véhémence en dénonçant notamment la notion de "droits collectifs " appliquée aux Québécois francophones en particulier, aux Autochtones et aux femmes ${ }^{28}$. En fait, le texte de l'Accord de Meech spécifiait que :

1. L'interprétation de la Constitution du Canada doit concorder avec

a) la reconnaissance que l'existence d'un Canada francophone, concentré mais non limité au Québec, et celle d'un Canada anglophone, concentré dans le reste du pays mais présent au Québec, constituent une caractéristique fondamentale de la fédération canadienne;

b) la reconnaissance que le Québec forme au sein du Canada une société distincte. 
2. Le Parlement et les législatures des provinces, dans l'exercice de leurs compétences respectives, prennent l'engagement de protéger la caractéristique fondamentale du Canada mentionnée au paragraphe (1)a).

3. L'Assemblée nationale et le gouvernement du Québec ont le rôle de protéger et de promouvoir le caractère distinct de la société québécoise mentionné au paragraphe (1)b $)^{29}$.

Sans aucun doute, par rapport au concept de " société distincte », définie différemment dans Charlottetown, Trudeau souleva quelques objections pertinentes et légitimes ${ }^{30}$. Par exemple :

S'agit-il de la collectivité canadiennefrançaise qui vit dispersée un peu partout au Canada? Évidemment non, puisque l'idéologie dominante au Québec se fiche éperdument du bilinguisme au Canada, et que devant les tribunaux elle prend fait et cause pour les gouvernements d'Alberta et de la Saskatchewan lorsque celles-ci suppriment les droits francophones acquis avant même l'entrée de ces provinces dans la Confédération en 1905. S'agit-il plutôt de la collectivité québécoise tout entière? Non plus, car cette « collectivité » s'appelle « province » et ses pouvoirs sont déjà reconnus explicitement par l'Acte constitutionnel de 1867. Il ne peut donc s'agir que d'une collectivité distincte à l'intérieur du Québec, mais laquelle encore? Certainement pas la collectivité anglophone, puisque la loi québécoise nie qu'elle puisse avoir des droits collectifs dans certains domaines : l'école et l'affichage, par exemple. Éliminons également les aborigènes, puisqu'on leur a clairement fait comprendre qu'ils ne pouvaient pas constituer une "société distincte » avec droit à l'auto-

29 Un dossier du Devoir, Le Québec et le lac Meech, Montréal, Guérin, 1987 à la p. 26.

30 Philippe Dubuisson, «L'entente de Charlottetown » La Presse (6 septembre 1992) A5. détermination, le vocable ayant déjà été réservé par des Québécois d'une autre race $^{31}$.

Ces questions étaient certainement légitimes. Toutefois par son insistance à opposer les droits collectifs aux droits individuels dans le cadre de ces débats sur l'Accord du lac Meech et l'Entente de Charlottetown, Pierre E. Trudeau a de nouveau contribué à répandre cette perception selon laquelle les Canadiens français du Québec sont « collectivistes ». Pourtant nous avons vu que les institutions de l'époque Trudeau sont elles-mêmes caractérisées par un équilibre entre des aspects collectifs et les droits individuels. La question est donc non pas d'opposer ces deux notions mais de trouver une solution qui permette un équilibre acceptable. Cet équilibre avait-il été pleinement atteint en 1982? Difficilement.

La première source de déséquilibre dans la $L o i$ constitutionnelle de 1982 tient donc au fait que la protection pour les droits linguistiques dans les articles 16 à 22 crée un espace de développement favorable au Canada français. Mais si la logique de l'argumentation tient au principe de l'opposition entre des droits collectifs et des droits individuels, pourquoi accorder au Canada français et, incidemment, à la collectivité canadienne-anglaise (après tout la langue anglaise est aussi protégée) une reconnaissance explicite dans les articles de la Charte portant sur les droits linguistiques.

Par conséquent, une politique fondée strictement sur le principe des droits individuels n'aurait-elle pas impliqué la seule énonciation de ces droits dans les articles 1 à 14 de la Charte et aucune référence aux droits linguistiques? Dans la mesure où, justement, les droits linguistiques, de même que les droits des Autochtones et le droit à l'égalité furent définis et inscrits dans la Charte, le Canada s'est doté d'institutions originales dans le contexte occidental. De plus, le processus menant à Charlottetown n'a fait qu'exprimer une culture politique fascinée par l'équilibre entre les communautés et les individus.

31 Pierre E. Trudeau, «Trudeau dit non : De la pauvreté de la pensée nationaliste au Québec» L'actualité 17:15 (1 octobre 1992) supp. I. 


\section{LES QUESTIONS IDENTITAIRES}

\section{LE DÉBAT AMÉRICAIN SUR LES IDENTITÉS}

Ces dernières remarques soulèvent toute la question de l'identité canadienne, notamment par rapport à l'identité politique américaine. Or, une approche importante dans l'étude des origines politiques des États-Unis fut celle de l'école dite du consensus. Élaborée par Louis Hartz dans les années 1950 pour l'étude des États-Unis, cette théorie fut reprise au Canada par des auteurs comme Kenneth McRae, Gad Horowitz, André J. Bélanger et, au moins implicitement, par Pierre E. Trudeau, en plus de susciter aux Etats-Unis même de nombreux débats ${ }^{32}$. Le concept clé de la théorie hartzienne est le « fragment idéologique »: au moment de la colonisation européenne du monde à partie du $\mathrm{XVI}^{\mathrm{e}}$ siècle, l'Europe aurait constitué un tout idéologique formé de deux principaux fragments : le fragment communautaire/féodal et le fragment individualiste. Ces deux fragments ont formé par la suite des points de repère dans un spectre idéologique composé, de gauche à droite, $\mathrm{du}$ socialisme, du libéralisme radical, du libéralisme « whig », du conservatisme « tory » et $\mathrm{du}$ féodalisme. Selon leur point d'origine et la situation idéologique particulière de leur métropole au moment de la colonisation, les sociétés nouvelles fondées aux États-Unis, au Canada, en Amérique latine et en Australie ont par conséquent développé une culture en fonction d'un de ces principaux fragments idéologiques. Par exemple, aux États-Unis, le fragment idéologique individualiste, associé à la pensée du philosophe John Locke (d'où l'utilisation de

32 Pour le Canada voir Peter J. Smith, «The Ideological Origins of Canada» (1987) 20 Canadian Journal of Political Science 3; Pour les Etats-Unis : Bernard Bailyn, The Ideological Origins of the American Revolution, Cambridge, The Belknap Press, 1967 et Gordon S. Wood, The Creation of the American Republic 1776-1787, Williamsburg, The University of North Carolina Press, 1969. La thèse opposée à celle de Hartz, fut:

J.G.A. Pocock, The Machiavellian Moment : Florentine Political Thought and the Atlantic Republican Tradition, Princeton, Princeton University Press, 1975. Pour la littérature plus récente, voir : Gordon S. Wood, «Rambunctious American Democracy» (2002) 49:8 The New York Review of Books 20; Robert A. Dahl, How Democratic Is the American Constitution?, New Haven, Yale University Press, 2001; et, Michael Novak, On Two Wings: Humble Faith and Common Sense at the American Founding, San Francisco, Encounter Books, 2002. l'expression « fragment lockien »), se serait imposé après la Révolution américaine au point de devenir la dimension essentielle de la société américaine. Au contraire, au Canada français et en Amérique latine, selon cette théorie, l'absence de vraies révolutions libérales aurait eu comme conséquence la perpétuation du fragment « féodal/ communautaire ». En d'autres mots, les sociétés coloniales d'origine anglo-saxonne, y compris le Canada anglais, seraient « lockiennes », les autres, dont le Canada français, seraient restées « féodales $»$.

Une autre école de pensée importante sur les origines politiques américaines fut celles inspirées de l'oeuvre de J.G.A. Pocock qui insista sur l'influence politique de la Renaissance italienne pour expliquer le radicalisme anglo-américain des $\mathrm{XVII}^{\mathrm{e}}$ et $\mathrm{XVIII}^{\mathrm{e}}$ siècles $^{33}$. Pocock utilisa l'expression d'humanisme civique pour décrire cette tradition atlantique au XVIII ${ }^{\mathrm{e}}$ siècle et la culture anglo-américaine. Cette tradition politique fut la principale forme d'opposition en GrandeBretagne aux nouvelles formes de propriété, de commerce et de finance amenées par la domination du parti Whig ou Court Party. L'opposition prit au contraire le nom de Country Party et s'attaqua à la corruption engendrée par le nouveau commerce. Ainsi, en Angleterre et dans les treize colonies de la Nouvelle-Angleterre, se développa une pensée politique opposée à la corruption créée par le commerce. Pour contrer cette influence négative, la tradition républicaine de l'humanisme civique s'inspira de l'œuvre de Machiavel sur l'Antiquité pour défendre l'idéal d'une société fondée sur la rigueur morale et la lutte contre la corruption. Thomas Jefferson aurait, dans le contexte américain, symbolisé cette tendance politique.

\section{LE DÉBAT CANADIEN INSPIRÉ DU DÉBAT AMÉRICAIN}

$\mathrm{Au}$ Canada, plus récemment, un auteur francophone important, Gérard Bouchard, a cherché à appréhender la dynamique de l'évolution identitaire des pays « neufs » en s'inspirant du

33 Pocock, ibid. 
débat sur les origines idéologiques ${ }^{34}$. Cette dynamique serait produite par deux éléments opposés, la continuité et la rupture, à partir desquels s'édifient les modèles $\mathrm{A}$ ou $\mathrm{B}$ de la genèse des pays neufs, c'est-à-dire les pays issus du colonialisme européen. Ainsi, le modèle A, selon Bouchard, est celui de la continuité et constitue une reproduction de la mère-patrie ou de la région-mère. Le discours identitaire au sein d'une société neuve qui émane du modèle A est celui de l'identique à la fois par rapport aux origines du pays neuf vis-à-vis de sa métropole mais aussi par rapport à leur destin commun. Au contraire, dans ce qu'il appelle le modèle $\mathrm{B}$, une collectivité neuve " tourne le dos " à la mèrepatrie et cherche à se reproduire dans la rupture et la différence. Certes, prévient Bouchard, il est impossible de trouver l'un ou l'autre de ces deux modèles à l'état pur. Ces modèles interagissent constamment et ne peuvent être conçus et appliqués que dans une perspective dialectique.

Le cas de l'Amérique latine fut à cet égard, selon Bouchard, très intéressant. De façon générale, l'évolution de l' Amérique latine depuis le $\mathrm{XVI}^{\mathrm{e}}$ siècle peut davantage être associée à la rupture plutôt qu'à la continuité. Mais le parcours ne fut pas linéaire. Après une première période " continuiste », les Créoles (Blancs nés en Amérique) en arrivèrent à développer une américanité opposée à la culture européenne. Au Mexique, comme ailleurs en Amérique latine, a écrit Bouchard, " il est remarquable que les plus anciennes expressions du sentiment de l'identité nationale incluaient l'indianité comme composante essentielle $»^{35}$.

La rupture avec les métropoles espagnoles et portugaises a été complétée au XIX ${ }^{\mathrm{e}}$ siècle lors des mouvements d'indépendance politique. Toutefois, plusieurs éléments de continuité ont perduré après l'acquisition de l'indépendance politique, notamment la langue, la religion et de nombreux emprunts à la culture savante européenne. La complexité sociale, culturelle et politique du continent a par la suite freiné l'élan de l'américanité et de l'indianité pour faire place à

34 Genèse des nations et cultures du Nouveau Monde, Montréal, Boréal, 2001.

35 Ibid. à la p. 195. l'échec du processus de rupture, voire à l'avènement d'une période de « réeuropéanisation », comme en Argentine et au Mexique sous le régime de Porfirio Diaz ${ }^{36}$.

Cela dit, selon Bouchard, la différenciation et la rupture restent des acquis de l'Amérique latine. L'américanité, certes, est inachevée et fragile mais réelle. L'acquisition de l'indépendance politique constitue une immense différence par rapport au Québec. Alors que l'Amérique latine entrait dans une période d'indépendance politique au $\mathrm{XIX}^{\mathrm{e}}$ siècle, le Québec, entre 1840 et 1940, donc après l'échec des Rébellions de 1837-1838, entrait au contraire dans une longue période continuiste. Ainsi, le Québec aurait traversé un certain nombre de périodes toutes marquées par le processus de continuité et de rupture. Par exemple, de 1608 à 1700, la Nouvelle-France aurait été une expérience de continuité, laquelle fut de plus en plus remise en question de 1700 à $1760^{37}$. La Conquête, bien sûr, a tout chambardé, créant une période de transition de 1760 à 1791 . Ce fut au cours de cette période de transition qu'une identité « canadienne » s'est formée au contact du colonialisme britannique, provoquant une période de rupture entre 1800 et 1838. L'échec des Rébellions en 1837-1838 provoqua cependant une fixation de l'identité dans le culturel et dans l'attachement aux origines françaises. S'en est suivie une période de remise en question à partir de 1940, voire de réaffirmation de l'objectif de rupture lors de la Révolution tranquille des années 1960. Dès lors, l'identité québécoise moderne serait potentiellement « vraie», puisque politique, par opposition à la « fausse identité canadiennefrançaise » fondée sur le culturel ${ }^{38}$. Mais cette « vraie » identité ne s'est pas encore complètement démarquée de l'ancienne. "L'identité nationale » n'est plus canadienne-française (à l'ancienne manière) mais elle n'est pas encore intégralement 
québécoise $^{39}$. Un peu plus loin, il écrit, « [1]e champ des allégeances est plus fragmenté que jamais. On dirait que le Québec est arrivé à un carrefour où toutes les fidélités, toutes les options, anciennes et nouvelles, se trouvent réunies : celles qu'il avait écartées, celles qu'il n'avait empruntées qu'à moitié et celles qu'a fait émerger son histoire récente $»^{40}$. En d'autres mots, pour développer son potentiel « humaniste civique » dans la tradition atlantique de reconnaissance des minorités et de développement d'un multiculturalisme francophone plus poussé qu'au Canada, le Québec devrait, selon Bouchard, rompre avec sa métropole politique, le Canada et à travers le Canada la Grande-Bretagne. Or le Québec a hésité à rompre. Est-ce la seule collectivité neuve dont l'avenir est hypothéqué par un lourd passé de continuité? Non. Trois exemples présentés par Bouchard montrent que la voie continuiste a été suivie par d'autres collectivités neuves. Ce sont l'Australie, la Nouvelle-Zélande, le Canada. Du XIX ${ }^{\mathrm{e}}$ siècle jusqu'aux trois dernières décennies du $\mathrm{XX}^{\mathrm{e}}$ siècle, ces trois collectivités neuves ne se sont pas affirmées en fonction de leur différence avec la mère-patrie mais plutôt en continuité. Trois facteurs ont formé cependant ces sociétés à se redéfinir : le déclin de l'Empire britannique après 1945, l'immigration massive et la combativité autochtone pour la reconnaissance notamment de leurs droits territoriaux. La pression de ces trois facteurs a profondément ébranlé l'identité continuiste dans ces trois collectivités. En cela, d'ailleurs, ces trois sociétés neuves se distinguent des États-Unis qui ont rompu dès 1776 avec la Grande-Bretagne.

En résumé, Bouchard, reprenant ici le modèle de Louis Hartz (1950), montre que les États-Unis ont développé une identité et une culture fondées sur l'individualisme et la liberté, rompant ainsi avec les mentalités collectives de la vieille Europe. Toutefois, il existe aussi un " autre » récit américain qui est celui des exclus de la société américaine, descendants d'esclaves, Autochtones, immigrants, qui ont été longtemps marginalisés par un discours national fondé sur la « mission

Ibid. à la p. 179.

40 Ibid. civilisatrice des Anglo-Saxons en Amérique ${ }^{41}$. Certes, Gérard Bouchard reconnaît cet aspect, mais conclut néanmoins que les États-Unis forment pleinement une société du nouveau monde, en rupture avec la vieille métropole.

Pour Bouchard, alors que les autres collectivités, malgré le caractère parfois inachevé de leur processus de rupture, ont réussi à obtenir leur indépendance politique, le Québec est la seule nation du nouveau monde, parmi les nations étudiées, à ne pas jouir de l'indépendance politique. C'est aussi la seule nation d'Amérique au XIX ${ }^{\mathrm{e}}$ siècle à s'être enfermée dans un discours unique et continuiste, et ce, de 1840 à 1940. En fait, la longue période continuiste de 1840 à 1940 a sans doute beaucoup contribué à retarder l'avènement au Québec d'une identité "vraie ", fondée sur l'objectif de rupture.

\section{CONCLUSION}

Dans la tradition des théories de Louis Hartz, reprise par Gérard Bouchard, les pays neufs, en particulier les États-Unis, auraient développé une tradition libérale fondée sur l'individualisme. Or le libéralisme est évidemment une doctrine complexe formée d'écoles de pensée parfois opposées. Ainsi, dans un essai sur le libéralisme, André Vachet a identifié les principales thèses (en l'occurrence la liberté, la sécurité, l'égalité et la propriété) et les principaux thèmes (rationalisme, naturalisme et individualisme) du libéralisme ${ }^{42}$.

La composante d'une forme de libéralisme par rapport à une autre tient à une différence de hiérarchie dans l'articulation de ces thèmes et thèses. Ainsi, le libéralisme dit classique qui est socialement conservateur accorde une priorité à l'articulation entre la propriété et l'individualisme. Mais au contraire, une autre tradition libérale insiste sur l'égalité, celle, notamment, de Rousseau, de Thomas Paine et de William Cobbett. Depuis deux siècles, la forme élitiste du libéralisme s'est souvent retrouvée dans les institutions des pays occidentaux. Au contraire, la forme plus égalitaire fut plus populaire et moins institutionnelle. $\mathrm{Si}$, comme l'ont soutenu

Ibid. à la p. 357.

L'idéologie libérale, Paris, Anthropos, 1970. 
récemment certains auteurs ${ }^{43}$, les institutions américaines ont été au départ antidémocratiques et élitistes et le sont demeurées jusqu'à aujourd'hui, par contraste, le Canada a développé des institutions qui reflètent à la fois l'élitisme et l'aspect progressiste d'un certain libéralisme. C'est ce que James Tully a appelé l'étrange multiplicité $\mathrm{du}$ Canada $^{44}$. Les étapes de cette étrange multiplicité ont marqué l'histoire du Canada mais aussi ses institutions. En ce sens, le bilinguisme et le multiculturalisme s'inscrivent d'abord et avant tout dans la résistance concrète de plusieurs groupes, dont les Canadiens français, peuple à la fois colonisateur et colonisé, à faire respecter leurs droits et dans la résistance de ces groupes non français et non britanniques d'origine à l'uniformité culturelle dans les institutions politiques. Une forme donc de libéralisme populaire de résistance a imposé des changements dans des institutions libérales. Ainsi, les circonstances de ces luttes ont permis d'inscrire la diversité et l'histoire au moins partiellement dans les institutions du pays et ce contrairement aux États-Unis où la constitution, et notamment le Bill of Rights, a fait peu de place, sinon aucune place, à la reconnaissance même partielle de la diversité. En ce sens, le modèle américain n'est pas nécessairement un modèle de rupture par rapport à l'élitisme de l'ancienne métropole. Son libéralisme est conservateur. Hartz et Bouchard ont donc tort, me semble-t-il. Le Canada, loin d'être un pays par définition continuiste, a au contraire des institutions, notamment issues de l'Acte de l'Amérique du Nord britannique en 1867 et de la Loi constitutionnelle de 1982 ou d'autres aspects, qui reflètent au moins partiellement la diversité, comme c'est le cas pour le bilinguisme et le multiculturalisme. En ce sens, le processus menant à Charlottetown et la clause Canada ont parfaitement reflété cette diversité et la richesse d'une autre tradition politique, unique au monde occidental, où toutes les dimensions sont liées.

\author{
Claude Couture \\ University of Alberta \\ Fullbright Scholar (2004-2005) \\ Henry M. Jackson School of International Studies, \\ University of Washington
}

\footnotetext{
43 Dubuisson, supra note 30

$44 \quad$ Supra note 24.
} 


\section{ANNEXE}

TeXte de L'Accord de ChaRLotTetown

\section{LES CITOYENS ET LES COLLECTIVITÉS}

\section{La clause Canada}

Il conviendrait d'incorporer en tant qu'article 2 de la Loi constitutionnelle de 1867 une nouvelle clause qui exprimerait les valeurs fondamentales du Canada. Cette disposition Canada guiderait les tribunaux dans leur interprétation de l'ensemble de la Constitution, y compris de la Charte canadienne des droits et libertés.

La Loi constitutionnelle de 1867 est modifiée par insertion, après l'article 1 , de ce qui suit :

2.(1) Toute interprétation de la Constitution du Canada, notamment de la Charte canadienne des droits et libertés, doit concorder avec les caractéristiques fondamentales suivantes :

a) le fait que le Canada est une démocratie attachée à un régime parlementaire et fédéral ainsi qu'à la primauté du droit;

b)le fait que les peuples autochtones du Canada, qui ont été les premiers gouvernants du territoire, ont le droit de promouvoir leurs langues, leurs cultures et leurs traditions et de veiller à l'intégrité de leurs sociétés, et le fait que leurs gouvernements forment un des trois ordres de gouvernements du pays;

c)le fait que le Québec forme au sein du Canada une société distincte, comprenant notamment une majorité d'expression française, une culture qui est unique et une tradition de droit civil;

d)l'attachement des Canadiens et de leurs gouvernements à l'épanouissement et au développement des communautés minoritaires de langue officielle dans tout le pays;

e)le fait que les Canadiens sont attachés à l'égalité raciale et ethnique dans une société qui comprend des citoyens d'origines multiples dont la contribution à l'édification d'un Canada fort reflète sa diversité culturelle et raciale;

f)l'attachement des Canadiens au respect des droits et libertés individuels et collectifs;

g)l'attachement des Canadiens au principe de l'égalité des personnes des deux sexes;

h)le fait que les Canadiens confirment le principe de l'égalité des provinces dans le respect de leur diversité.

(2) La législature et le gouvernement du Québec ont le rôle de protéger et de promouvoir la société distincte. 